\title{
Elementos minerais em folhas de espécies arbóreas de Mata Atlântica e Mata de Restinga, na Ilha do Cardoso, $\mathbf{S P}^{1}$
}

\author{
REGINA M. DE MORAES ${ }^{2}$ e MARISA DOMINGOS ${ }^{2}$
}

(recebido em 22/06/95; aceito em 20/12/96)

\begin{abstract}
Mineral elements in leaves of tree specie from Atlantic and "Restinga" Forests, at Ilha do Cardoso, SP). In continuity to the study of the nutrient cycling process in the Atlantic and "Restinga" Forests at Ilha do Cardoso ( $25^{\circ} 12^{\prime}$ S and $\left.48^{\circ} 01^{\prime} \mathrm{W}\right)$, São Paulo, the concentrations of macro and micronutrients, $\mathrm{Na}$ and $\mathrm{Al}$ in the leaf compartment, represented by the most important tree species, are presented. The 10 species with the highest importance value index were included, whose leaves composed mixed samples. Samples were taken in Jun/93 and Dez/93. The contents of all elements, excluding B and Na, were higher in leaves from the Atlantic Forest. The concentrations of most of the minerals were higher in the fresh leaves than in the leaf litterfall, becoming evident the occurrence of strategies of nutrient economy in both forests, especially at the "restinga", indicating its high useefficiency of a small nutrient standing stock.
\end{abstract}

RESUMO - (Elementos minerais em folhas de espécies arbóreas de Mata Atlântica e Mata de Restinga, na Ilha do Cardoso, SP). Os teores de macro e micronutrientes, $\mathrm{Na}$ e $\mathrm{Al}$ foram determinados no compartimento das folhas, representado pelas espécies arbóreas mais importantes ocorrentes em áreas cobertas com Mata Atântica e Mata de Restinga, na Ilha do Cardoso ( $25^{\circ} 12^{\prime}$ 'S e $\left.48^{\circ} 01^{\prime} \mathrm{W}\right)$, São Paulo, como parte de um estudo sobre ciclagem de nutrientes. Utilizaram-se, em cada área, as 10 espécies com maiores índices de valor de importância, cujas folhas compuseram amostras mistas, realizando-se duas coletas (jun/ 93 e dez/93). Todos os elementos analisados apresentaram concentrações mais elevadas nas folhas coletadas na Mata Atlântica, com exceção de B e Na. A concentração da maioria dos elementos minerais foi mais alta nas folhas vivas do que na serapilheira foliar produzida em cada ambiente, sendo evidenciadas estratégias de economia de nutrientes, em ambas as formações vegetais, porém mais acentuadas na Mata de Restinga, indicando sua alta eficiência na utilização de um pequeno estoque de nutrientes.

Key words - Leaf nutrient, mineral cycling, Atlantic Forest, "Restinga" Forest

\section{Introdução}

A análise foliar é uma prática tradicional nos estudos sobre nutrição mineral (Grubb 1977) e, entre outros aspectos, tem sido empregada na classificação de florestas (Klinge et al. 1983, Klinge 1985) e no estudo da relação entre características morfológicas e anatômicas e oligotrofismo (Sobrado \& Medina 1980, Medina et al. 1990). A concentração de elementos minerais em tecidos foliares pode refletir a fertilidade dos solos, sendo que os dados relativos a este parâmetro são úteis para a comparação da condição nutricional de diferentes áreas (Vitousek \& Sanford 1986).

Dentro desta concepção, e buscando maior compreensão dos processos relativos à ciclagem de nutrientes, que contribuem para o estabelecimento de diferentes tipos de vegetação sobre diferentes

1. Trabalho realizado dentro do Programa Linhas de Ação em Botânica do CNPq, com o apoio do Convênio 11/94 Subcomponente Mata Atlântica/BIRD/MMA-PNMA/ SMA-SP.

2. Seção de Ecologia, Instituto de Botânica, Caixa Postal 4005, 01061-970 São Paulo, SP, Brasil. tipos de solos, realizou-se um estudo integrado das diversas etapas do processo de ciclagem de elementos minerais em duas formações vegetais, Mata Atlântica e Mata de Restinga, situadas na Ilha do Cardoso, estado de São Paulo, tomando-se por base o modelo de compartimentos e fluxos proposto por Eriksson (1971), Moraes (1993), Rebelo (1994) e Varjabedian (1994) apresentaram resultados de produção e decomposição de serapilheira e entrada de nutrientes via precipitação. No presente trabalho, são mostrados os teores de macro e micronutrientes, $\mathrm{Na}$ e Al presentes no compartimento das folhas, aqui representado pelas espécies arbóreas mais importantes de ambas as formações, relacionando-os à fertilidade do solo, à eficiência de sua utilização e aos aspectos sazonais.

Estudos dessa natureza, em ecossistemas preservados, como os da Ilha do Cardoso, são importantes como base para o entendimento das alterações do processo de ciclagem de nutrientes causadas por interferências antrópicas em ecossistemas similares e como subsídios para a sua recuperação. Este é especialmente o caso das áreas cobertas por Mata Atlântica e da vegetação sobre restingas do sudeste brasileiro. 


\section{Material e métodos}

A área de estudos, Ilha do Cardoso $\left(25^{\circ} 03^{\prime} \mathrm{S}, 48^{\circ} 01^{\prime} \mathrm{W}\right)$, situa-se ao sul do estado de São Paulo no município de Cananéia. Apresenta clima super úmido, mesotérmico, sem estação seca definida (Funari et al. 1987), com precipitação média anual de $2300 \mathrm{~mm}$. Os meses com maior pluviosidade são janeiro, fevereiro e março. A temperatura média anual é de $21^{\circ} \mathrm{C}$, sendo os meses mais quentes janeiro e fevereiro. Seu relevo caracterizase pela presença de um grande maciço montanhoso central, coberto por Mata Atlântica, o qual é cercado por planícies sedimentares, apresentando restingas e manguesais nas áreas voltadas para o oceano e para a zona lagunar, respectivamente.

Os sítios experimentais ficam a NE da ilha em áreas de Mata Atlântica, a 120 m.s.m., e de Mata de Restinga, na planície litorânea, sobre solos de moderada e baixa fertilidade respectivamente (tabela 1 ).

Visando obter melhor aproximação às concentrações médias existentes no compartimento de folhas do ecossistema, bem como compará-las às determinadas em outros compartimentos, amostras mistas foram compostas utilizando-se folhas das 10 espécies arbóreas com os maiores índices de valor de importância (IVI) em cada área (tabela 2). A seleção das espécies amostradas foi baseada em levantamentos fitossociológicos prévios realizados por Sugyiama (1993) e Melo \& Mantovani (1994).

Assumiu-se que as espécies mais importantes absorvem maior quantidade de nutrientes, produzem e derrubam mais folhas e, conseqüentemente, têm maior participação no processo de ciclagem de nutrientes do que as menos importantes. Assim sendo, para a composição de cada amostra mista, foram utilizadas quantidades proporcionais de folhas de cada espécie, em gramas de massa seca, sendo colocadas dez partes $(10 \mathrm{~g})$ daquela com o maior IVI, nove partes $(9 \mathrm{~g})$ daquela com segundo maior IVI e assim sucessivamente até a colocação de uma parte $(1 \mathrm{~g})$ da espécie com o décimo maior valor do índice de importância.

Foram realizadas duas coletas, em junho/93 (estação menos chuvosa) e dezembro/93 (estação mais chuvosa), para a verificação de possíveis variações sazonais.

Em cada coleta e em cada floresta, foram recolhidas folhas de três individuos de cada espécie, os quais foram sorteados entre os indivíduos incluídos nos levantamentos fitossociológicos. No laboratório, cada amostra mista foi composta com folhas de um indivíduo por espécie, utilizando-se as quantidades de massa seca descritas acima, de forma a serem obtidas três amostras por área e por período de coleta. Essas amostras foram analisadas quanto aos teores de macro e micronutrientes, sódio e alumínio pelo Laboratório de Química Analítica do CENAUSP (Piracicaba), segundo Zagatto et al. (1981).
Utilizou-se o teste não paramétrico de Wilcoxon (Campos 1982) para a comparação dos resultados obtidos nos diferentes períodos de coleta e áreas de estudo.

Adicionalmente, os valores médios das concentrações dos elementos analisados foram comparados aos obtidos no estudo da produção de serapilheira realizado nas mesmas áreas por Moraes (1993), estimando-se a porcentagem de translocação de nutrientes nas folhas antes de sua derrubada, segundo a fórmula proposta por Vitousek \& Sanford (1986), em que o quociente entre as concentrações dos nutrientes e do cálcio na serapilheira foi dividido pelo mesmo quociente nas folhas vivas.

\section{Resultados e Discussão}

Nos dois períodos de coleta, as concentrações de todos os elementos analisados foram mais altas nas folhas de árvores da Mata Atlântica, exceto quanto a $\mathrm{B}$ e $\mathrm{Na}$, que foram superiores na restinga (tabela 3). Todas estas diferenças foram comprovadas estatisticamente $(p<0,05)$. Nitrogênio foi o elemento presente em maiores proporções em todas as amostras. As diferenças mais pronunciadas entre as duas áreas relacionaram-se aos elementos $\mathrm{Al}, \mathrm{Mn}, \mathrm{K}$ e N, cujas concentrações foram, respectivamente, $16,8,3$ e 2,4 vezes mais altas nas folhas provenientes de Mata Atlântica.

Vitousek \& Sanford (1986) sumarizaram dados sobre concentração foliar de nutrientes em 18 florestas tropicais, agrupando-as de acordo com a fertilidade do solo. Os resultados obtidos na Mata de Restinga aproximaram-se daqueles apresentados nas florestas sobre solos de baixa fertilidade, classificados no grupo dos podzóis. Já as concentrações de $\mathrm{K}, \mathrm{Ca}$ e $\mathrm{Mg}$ verificadas nas folhas da Mata Atlântica aproximaram-se das obtidas nas florestas sobre solos moderadamente férteis, enquanto as de $\mathrm{P}$ foram inferiores. $\mathrm{O}$ teor de $\mathrm{N}$ na Mata Atlântica da Ilha do Cardoso, por outro lado, foi mais alto não apenas em relação à média, mas

Tabela 1. Características dos solos das áreas de estudo, Ilha do Cardoso, Cananéia. SP. Textura classificada segundo EMBRAPA (1979). $\mathrm{S}=$ soma de bases; $\mathrm{CTC}=$ capacidade de troca catiônica; V = saturação de bases. Dados apresentados em Moraes (1993).

\begin{tabular}{|c|c|c|c|c|c|c|c|c|}
\hline & $\begin{array}{l}\text { Profundidade } \\
\text { (cm) }\end{array}$ & Textura & $\begin{array}{c}\mathrm{pH} \mathrm{em} \\
\mathrm{H}_{2} \mathrm{O}\end{array}$ & $\mathrm{C} / \mathrm{N}$ & $\begin{array}{c}\text { M.O. } \\
(\%)\end{array}$ & $\mathrm{S}$ & CTC & $\begin{array}{c}\mathrm{V} \\
(\%)\end{array}$ \\
\hline \multirow[t]{3}{*}{ Mata Atlântica } & $0-15$ & franco-argilo-arenoso & 3,2 & 11,1 & 5,92 & 1,62 & 4,71 & 34,4 \\
\hline & $15-30$ & franco-argiloso & 4,2 & 11,3 & 3,51 & 0,78 & 3,70 & 21,1 \\
\hline & $30-50$ & franco argiloso & 3,6 & 9,6 & 1,98 & 0,57 & 3,67 & 15,5 \\
\hline \multirow[t]{2}{*}{ Mata de Restinga } & $0-15$ & arenoso & 3,0 & 22,0 & 3,03 & 0,50 & 1,74 & 28,7 \\
\hline & $15-30$ & arenoso & 3,4 & 12,0 & 0,21 & 0,05 & 0,51 & 9,8 \\
\hline
\end{tabular}


Tabela 2. Espécies utilizadas para a composição de amostras mistas de folhas de espécies arbóreas da Mata Atlântica e Mata de Restinga, Ilha do Cardoso, Cananéia, SP, relacionadas em ordem decrescente de IVI. Dados apresentados em Sugyiama (1993) e Melo \& Mantovani (1994).

\begin{tabular}{l}
\hline \multicolumn{1}{c}{ Espécie } \\
\hline Mala Atlântica \\
1. Euterpe edulis Mart. \\
2. Psychotria nuda (Cham.) Wawra \\
3. Rudgea jasminoides (Cham.) M. Arg. \\
4. Cryptocarya moschata Nees \& Marl. ex Nees \\
5. Malouetia cestroides (Nees \& Mart.) M. Arg. \\
6. Virola oleifera (Scott) A.C. Smith \\
7. Rheedia gardneriana Planch \& Triana \\
8. Chrysophyllum flexuosum Mart. \\
9. Astrocaryum aculeatissimum (Scott) Burret \\
10. Calyptrantes lucida Mart. \\
Mata de Restinga \\
1. Ternstroemia brasiliensis Camb. \\
2. Ocotea pulchella (Nees) Mez \\
3. Clusia criuva Camb. \\
4. Ilex theezans var. acrodonta Loes \\
5. Ilex theezans var. grandifolia Loes \\
6. Myrcia multiflora (Lam.) D.C. \\
7. Weinmannia pauliniifolia Pohl \\
8. Gomidesia fenzliana Berg. \\
9. Andira fraxinifolia Benth. \\
10. Ilex dumosa Reissek \\
\hline
\end{tabular}

também ao limite superior da faixa apresentada por aqueles autores e aos valores obtidos em florestas tropicais e incluídos em listagem organizada por Klinge (1985).

A concentração de nitrogênio expressa em porcentagem de massa seca está relacionada em grande parte à anatomia foliar, pois depende da proporção entre material das paredes celulares, pobre em N, e citoplasma, rico em N (Grubb 1977, Vitousek \& Sanford 1986). Espécies que apresentam características de escleromorfismo, como muitas das ocorrentes na Mata de Restinga, tendem a apresentar concentrações de $\mathrm{N}$ baixas. Deste modo, as diferenças nos níveis de $\mathrm{N}$ entre os dois tipos de mata estudados podem estar associadas não somente às diferenças da fertilidade do solo (tabela 1) como também à maior presença de espécies com características de escleromorfismo na Mata de Restinga.
A alta concentração de $\mathrm{Al}\left(5000 \mu \mathrm{g} \cdot \mathrm{g}^{-1}\right)$ observada no compartimento de folhas da Mata Atlântica pode indicar a existência de espécies acumuladoras na comunidade vegetal, considerando como tais aquelas com níveis foliares superiores a $1000 \mu \mathrm{g} \cdot \mathrm{g}^{-1}$, de acordo com definição proposta por Weeb (1954, apud Sobrado \& Medina 1980), fato que deve estar refletindo as altas concentrações do elemento no solo desta área.

$\mathrm{O}$ teor de $\mathrm{Na}$, bastante elevado nas duas áreas, pode ser atribuído ao efeito da proximidade do mar.

Com relação à sazonalidade, verificou-se que, na Mata Atlântica, N, Mn e Zn apresentaram-se em concentrações mais elevadas em junho, e K, Cu e Na, em dezembro $(\mathrm{p}<0,05)$. Na Mata de Restinga, os níveis de $\mathrm{K}, \mathrm{Fe}, \mathrm{Zn}, \mathrm{Al}$ e $\mathrm{B}$ foram mais altos em dezembro, não se verificando diferenças nos teores dos outros elementos entre os períodos de coleta. Britez (1994) verificou que, em Mata de Restinga no Paraná, as concentrações de $\mathrm{K}, \mathrm{Ca}$ e $\mathrm{Mg}$ também foram mais altas em dezembro, período de maior produção de serapilheira. Segundo esse autor, tal fato pode estar relacionado às condições ambientais
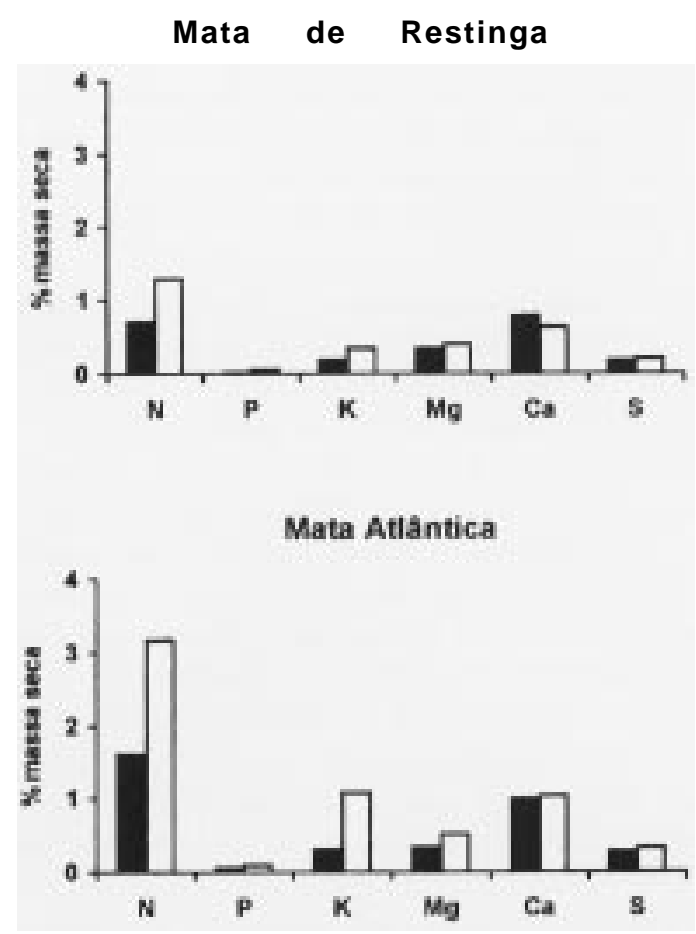

Figura 1. Concentrações de macronutrientes (\% de massa seca) na serapilheira foliar ( $\square$ ) e em amostras de folhas vivas $(\square)$ de espécies arbóreas ocorrentes em cada uma das áreas de estudo, Ilha do Cardoso, Cananéia, SP. 
Tabela 3. Médias e respectivos desvios padrão das concentrações de macronutrientes (\% de massa seca), micronutrientes, $\mathrm{Na}$ e $\mathrm{Al}$ ( $\mu \mathrm{g}$. $\mathrm{g}^{-1}$ de massa seca) em amostras mistas de folhas de espécies arbóreas da Mata Atlântica e Mata de Restinga, Ilha do Cardoso, Cananéia, SP.

\begin{tabular}{|c|c|c|c|c|c|c|}
\hline \multirow[t]{2}{*}{ Nutrientes } & \multicolumn{3}{|c|}{ Mata Atlântica } & \multicolumn{3}{|c|}{ Mata de Restinga } \\
\hline & Junho & Dezembro & Média anual & Junho & Dezembro & Média anual \\
\hline \multicolumn{7}{|l|}{$\%$} \\
\hline $\mathrm{N}$ & $3,38 \pm 0,16$ & $2,95 \pm 0,19$ & $3,17 \pm 0,26$ & $1,29 \pm 0,04$ & $1,30 \pm 0,03$ & $1,30 \pm 0,03$ \\
\hline $\mathrm{P}$ & $0,10 \pm 0,01$ & $0,10 \pm 0,01$ & $0,10 \pm 0,01$ & $0,05 \pm 0,01$ & $0,06 \pm 0,01$ & $0,06 \pm 0,01$ \\
\hline K & $0,98 \pm 0,03$ & $1,17 \pm 0,09$ & $1,08 \pm 0,11$ & $0,33 \pm 0,03$ & $0,37 \pm 0,01$ & $0,35 \pm 0,03$ \\
\hline $\mathrm{Ca}$ & $1,03 \pm 0,14$ & $1,03 \pm 0,04$ & $1,03 \pm 0,09$ & $0,60 \pm 0,07$ & $0,66 \pm 0,02$ & $0,63 \pm 0,05$ \\
\hline $\mathrm{Mg}$ & $0,49 \pm 0,05$ & $0,50 \pm 0,02$ & $0,47 \pm 0,05$ & $0,38 \pm 0,05$ & $0,41 \pm 0,01$ & $0,40 \pm 0,03$ \\
\hline $\mathrm{S}$ & $0,33 \pm 0,02$ & $0,32 \pm 0,02$ & $0,33 \pm 0,02$ & $0,19 \pm 0,01$ & $0,21 \pm 0,01$ & $0,20 \pm 0,02$ \\
\hline \multicolumn{7}{|l|}{$\mu \mathrm{g} \cdot \mathrm{g}^{-1}$} \\
\hline $\mathrm{Fe}$ & $141 \pm 16$ & $145 \pm 17$ & $143 \pm 14$ & $81 \pm 5$ & $122 \pm 5$ & $101 \pm 21$ \\
\hline $\mathrm{Cu}$ & $11 \pm 1$ & $13 \pm 1$ & $12 \pm 1$ & $6 \pm 1$ & $6 \pm 2$ & $6 \pm 1$ \\
\hline $\mathrm{Zn}$ & $42 \pm 3$ & $37 \pm 2$ & $39 \pm 3$ & $23 \pm 2$ & $25 \pm 2$ & $24 \pm 2$ \\
\hline $\mathrm{Mn}$ & $1915 \pm 317$ & $1073 \pm 221$ & $1494 \pm 476$ & $197 \pm 32$ & $194 \pm 29$ & $196 \pm 25$ \\
\hline B & $35 \pm 6$ & $37 \pm 3$ & $36 \pm 4$ & $46 \pm 3$ & $52 \pm 1$ & $49 \pm 4$ \\
\hline $\mathrm{Al}$ & $4675 \pm 1313$ & $5381 \pm 614$ & $5027 \pm 909$ & $266 \pm 76$ & $374 \pm 26$ & $320 \pm 71$ \\
\hline $\mathrm{Na}$ & $2600 \pm 400$ & $3500 \pm 300$ & $3017 \pm 543$ & $4100 \pm 300$ & $4067 \pm 100$ & $4083 \pm 157$ \\
\hline
\end{tabular}

mais favoráveis à decomposição neste período do ano, que possibilitam maior disponibilidade de nutrientes à vegetação. Os resultados apresentados aqui, entretanto, não permitem a visualização nítida de tendências ou padrões sazonais.

Medina et al. (1990) discutiram o quociente P/N em folhas vivas de espécies das florestas de San Carlos, Amazônia venezuelana. Obtiveram um valor de 0,05 e concluíram que o fósforo está presente sempre em pequenos suprimentos nestes ambientes. $\mathrm{Na}$ Ilha de Maracá (RO), Thompson et al. (1992) encontraram uma taxa $\mathrm{P} / \mathrm{N}$ de 0,063 , sugerindo maior disponibilidade de fósforo na área de estudo. $\mathrm{Na}$ Mata Atlântica da Ilha do Cardoso, esta taxa foi de 0,032 e na Mata de Restinga, 0,042, valores consideravelmente baixos que indicam pequena disponibilidade de $\mathrm{P}$ nestes ambientes. As concentrações de $\mathrm{P}$ em folhas da Mata Atlântica foram mais altas do que as encontradas nas folhas da Mata de Restinga, mas o quociente P/N foi menor porque as diferenças nas concentrações de $\mathrm{N}$ foram mais acentuadas. Não foi verificada correlação entre as concentrações desses dois elementos, ao contrário do relatado por Sobrado \& Medina (1980) e Medina et al. (1990). Estes resultados reforçam os comentários de Thompson et al. (1992) que consideram de valor limitado o emprego deste quociente como um indicador ecológico do suprimento de P e de Medina et al. (1990), segundo os quais ele reflete mais as adaptações fisiológicas das espécies a condições a longo prazo do que a disponibilidade de $\mathrm{P}$ em um dado momento.

A concentração da maioria dos macronutrientes foi mais alta nas folhas vivas do que na serapilheira foliar recém produzida (figuras 1 e 2), em cada ambiente, mostrando haver translocação de elementos como N, P, Mg e S (tabela 4) e evidenciando estratégias de economia de nutrientes em ambas as florestas. Esse processo é mais intenso na comunidade vegetal da restinga, a qual mostra uma grande adaptação à escassez de nutrientes, os

Tabela 4. Translocação de nutrientes minerais em folhas da Mata Atlântica e Mata de Restinga, Ilha do Cardoso, Cananéia, SP. estimada de acordo com Vitousek \& Sanford 1986.

\begin{tabular}{ccc}
\hline Elemento & \multicolumn{2}{c}{ Percentuais translocados } \\
& Mata Atlântica & Mata de Restinga \\
\hline $\mathrm{N}$ & 47 & 57 \\
$\mathrm{P}$ & 47 & 74 \\
$\mathrm{Mg}$ & 27 & 31 \\
$\mathrm{~K}$ & 71 & 59 \\
$\mathrm{~S}$ & 12 & 32 \\
\hline
\end{tabular}


quais são reaproveitados ao participarem da ciclagem interna do ecossistema. Tal fato é bem ilustrado pela alta porcentagem de translocação de $\mathrm{P}(74 \%)$. Estes resultados confirmam que os mecanismos de translocação são mais efetivos em ecossistemas oligotróficos, como proposto por diversos autores (Grubb 1977, Chapim III 1980, Vitousek \& Sanford 1986).

A concentração de $\mathrm{K}$ na serapilheira foliar também foi menor do que nas folhas vivas, em ambas as florestas. Este elemento apresenta grande mobilidade por não ser estrutural, sendo facilmente retirado por translocação das folhas senescentes e por lixiviação antes e após a senescência (Vitousek \& Sanford 1986). O fato de a perda deste íon ter sido mais acentuada na Mata Atlântica $(79 \%)$ pode estar associado à maior precipitação anual registrada neste ambiente, $2600 \mathrm{~mm}$ do que na Mata de Restinga, 1700 mm (Varjabedian 1994), possivelmente intensificando o processo de lixiviação.
Segundo Vitousek \& Sanford (1986), florestas produtivas são ricas em $\mathrm{P}, \mathrm{K}$ e $\mathrm{Ca}$, ciclam grandes quantidades de nutrientes e desenvolvem-se sobre solos comparativamente férteis, enquanto em áreas menos férteis, desenvolvem-se florestas menos produtivas, as quais contêm e ciclam menores quantidades de nutrientes. Assim, as características químicas do solo e a qualidade da água em solução são fatores capazes de governar as condições nutricionais da vegetação (Klinge et al. 1983). No presente estudo, essas afirmações pareceram se confirmar, visto que a Mata Atlântica, com maior disponibilidade de nutrientes, apresentou uma folhagem quimicamente mais rica que a do ambiente da restinga. Também em comparação com outras florestas tropicais, as concentrações nas folhas da Mata Atlântica são altas, principalmente quanto a $\mathrm{N}$, enquanto as verificadas nas folhas de espécies da Mata de Restinga assemelham-se às obtidas em formações que ocorrem em regiões oligotróficas relacionadas por Klinge (1985), tais como campina
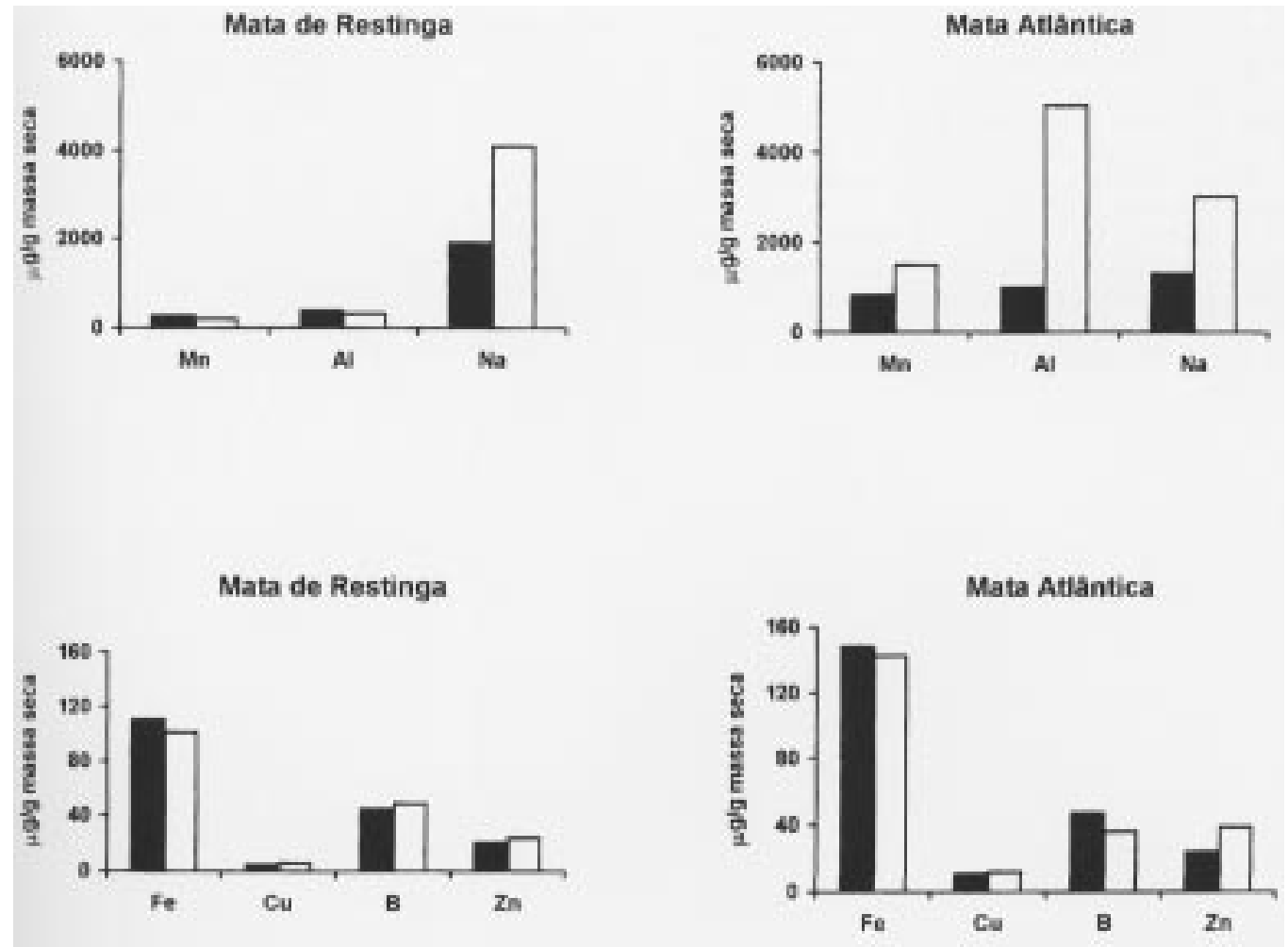

Figura 2. Concentrações de micronutrientes, $\mathrm{Na}$ e $\mathrm{Al}\left(\mu \mathrm{g} \cdot \mathrm{g}^{-1}\right)$ na serapilheira foliar $(\square)$ e em amostras de folhas vivas $(\square)$ de espécies arbóreas ocorrentes em cada uma das áreas de estudo, Ilha do Cardoso, Cananéia, SP. 
amazônica, mata de igapó, caatinga e savana. Os baixos níveis de nutrientes no compartimento de folhas deste ecossistema, juntamente com as altas porcentagens de translocação, indicam sua alta eficiência na utilização de um pequeno estoque de nutrientes, conforme mencionado em Moraes (1993).

Agradecimentos - À Maria Margarida R.F. Melo e à Marie Sugyiama, do Instituto de Botânica, pelo fornecimento de dados de campo que, em muito, auxiliaram a execução deste trabalho.

\section{Referências bibliográficas}

BRITEZ, R.M. 1994. Ciclagem de nutrientes minerais em duas florestas da planície litorânea da Ilha do Mel, Paranaguá, PR. Dissertação de mestrado, Universidade Federal do Paraná, Paraná.

CAMPOS, H. 1982. Estatística experimental não paramétrica. EDUSP, Piracicaba.

CHAPIM, F.S. III 1980. The mineral nutrition of wild plants. Ann. Rev. Ecol. Syst. 11:233-260.

EMBRAPA, 1979. Manual de métodos de análise de solo. Serviço Nacional de levantamento e conservação de solos, Rio de Janeiro.

ERIKSSON, E. 1971. Compartment models and the reservoir theory. Ann. Rev. Ecol. Syst. 2:67-83.

FUNARI, F.L., DE VUONO, Y.S. \& SALUM, S.T. 1987. Balanço hídrico de duas áreas de Mata Atlântica: Reserva Biológica de Paranapiacaba e Parque Estadual da Ilha do Cardoso (estado de São Paulo). In Anais do IV Congresso da Sociedade Botânica de São Paulo, p.95-101.

GRUBB, P.J. 1977. Control of forest growth and distribution on wet tropical mountais with special reference to mineral nutrition. Ann. Rev. Ecol. Syst. 8:83-107.

KLINGE, H., FURCH, K., HARMS, E. \& REVILLA, J. 1983. Foliar nutrient levels of native tree species from Central Amazonia. 1. Inundation forests. Amazoniana 8:19-45.

KLINGE, H. 1985. Foliar nutrient levels of native trees species from Central Amazonia. 2. Campina. Amazoniana 9:281-295
MEDINA, E., GARCIA, V. \& CUENCA, E. 1990. Sclerophylly and oligotrophic environments: relationships between leaf structure, mineral nutrient content, and drought resistance in tropical rain forests of the upper rio Negro region. Biotropica 22:51-64.

MELO, M.M.R.F. \& MANTOVANI, W. 1994. Composição florística e estrutura de trecho de Mata Atlântica de encosta na Ilha do Cardoso (Cananéia, SP, Brasil). Bolm. Inst. Bot. 9:107-158.

MORAES, R.M. 1993. Ciclagem de nutrientes minerais em Mata Atlântica de encosta e Mata sobre Restinga, na Ilha do Cardoso, Cananéia, SP: Produção de serapilheira e transferência de nutrientes. Dissertação de mestrado, Universidade de São Paulo, São Paulo.

REBELO, C.F. 1994. Ciclagem de nutrientes minerais em floresta pluvial tropical de encosta e em floresta sobre restinga na Ilha do Cardoso, Cananéia, SP: decomposição da serapilheira. Dissertação de mestrado, Universidade de São Paulo, São Paulo.

SOBRADO, M.A. \& MEDINA, E. 1980. General morphology, anatomical structure, and nutrient content of sclerophyllous leaves of the "Bana" vegetation of Amazonas. Oecologia 45:341-345.

SUGYIAMA, M. 1993. Estudo de florestas na restinga da Ilha do Cardoso, Cananéia, SP. Dissertação de mestrado, Universidade de São Paulo, São Paulo.

THOMPSON, J., PROCTOR, J., VIANA, V., MILLIKEN, W., RATTER, J.A. \& SCOTT, D.A. 1992. Ecological studies on a lowland evergreen rain forest on Maracá Island, Roraima, Brazil. I. Physical environment, forest structure and leaf chemistry. J. Ecol. 80:689-703.

VARJABEDIAN, R. 1994. Aspectos comparativos da ciclagem de nutrients minerais em Mata Atlântica da encosta e em Mata sobre Restinga, no Parque Estadual da Ilha do Cardoso, SP. Dissertação de mestrado, Universidade de São Paulo, São Paulo.

VITOUSEK, P.M. \& SANFORD, R.L.Jr. 1986. Nutrient cycling in moist tropical forest. Ann. Rev. Ecol. Syst. 17:137-167.

ZAGATTO, E.A.G., JACINTHO, A.O., REIS, B.F., KRUG, F.J., BERGAMIN FILHO, H., PESSENDA, L.C.R., MORTATTI, J \& GINÉ, M.F. 1981. Manual de análise de plantas e águas empregando sistemas de injeção em fluxo. CENA/USP, Piracicaba. 